\title{
Acid Exposure Time $>6 \%$ Might Not Improve the Therapeutic Outcome in Chinese Gastroesophageal Reflux Disease Patients
}

\author{
Yuqing Lin, ${ }^{1}$ Yuwen Li, ${ }^{1}$ Mengya Liang, ${ }^{2}$ Niandi Tan, ${ }^{1}$ Mengyu Zhang, ${ }^{1}$ Songfeng Chen, ${ }^{1}$ and Yinglian Xiao ${ }^{1 *}$ \\ Departments of ${ }^{1}$ Gastroenterology and Hepatology, and ${ }^{2}$ Cardiac Surgery, the First Affiliated Hospital, Sun Yat-sen University, Guangzhou, China
}

\begin{abstract}
Background/Aims
There is less acid burden in Chinese gastroesophageal reflux disease (GERD) patients. However, the Lyon consensus proposed a higher threshold of acid exposure time (AET > 6\%) for GERD. The aims are to apply the updated criteria in Chinese GERD patients and clarify its influence on clinical outcome.
\end{abstract}

\section{Methods}

Patients who were referred for both esophageal high-resolution manometry and 24-hour esophageal pH monitoring due to reflux symptoms were retrospectively screened. Those patients with AET $>4 \%$ was included and grouped into either AET 4-6\% or AET $>6 \%$. Their manometric profile, reflux profile, and response to proton pump inhibitors (PPIs) were evaluated. Adjunctive evidence proposed in the Lyon consensus was added in patients with AET 4-6\% for therapeutic gain. Another group of patients $(n=144)$ with AET $<4 \%$ were included as non-GERD patients.

\section{Results}

In total, 151 patients (102 males) were included with 113 patients AET > 6\% (74.9\%). GERD patients with AET > 4\% were with more male, older patients, and higher body mass index compared with non-GERD patients. Meanwhile, GERD patients were less competent in esophagogastric junction pressure. However, the manometric and reflux profile were similar between patients with AET $>6 \%$ and 4-6\%. The response rate of PPI therapy was $64.6 \%$ and $63.2 \%$, respectively, in groups of AET $>6 \%$ and $4-6 \%(P>0.05)$. When adjunctive evidence was added in patients with AET 4-6\%, no therapeutic gain was obtained.

\section{Conclusions}

The efficacy of PPI therapy was similar in patients with AET $>6 \%$ and 4-6\%. The increase of the AET threshold did not influence the clinical outcome of Chinese GERD patients.

(J Neurogastroenterol Motil 2021;27:55-62)

Key Words

Esophageal pH monitoring; Gastroesophageal reflux; Proton pump inhibitors

Received: November 19, 2019 Revised: March 5, 2020 Accepted: March 22, 2020

() This is an Open Access article distributed under the terms of the Creative Commons Attribution Non-Commercial License (http://creativecommons. org/licenses/by-nc/4.0) which permits unrestricted non-commercial use, distribution, and reproduction in any medium, provided the original work is properly cited.

*Correspondence: Yinglian Xiao, MD, PhD Department of Gastroenterology and Hepatology, The First Affiliated Hospital, Sun Yat-sen University, 58 Zhongshan Road 2, Guangzhou 510080, China Tel: +86-20-84113181, Fax: +86-20-87332916, E-mail: xyingl@mail.sysu.edu.cn 


\section{Introduction}

Gastroesophageal reflux disease (GERD) is a symptom-based diagnosis. ${ }^{1-3}$ However, heartburn is a heterogeneous symptom and it could be the primary symptom of both GERD and non-GERD, including functional esophageal disorders and achalasia. Although proton pump inhibitors (PPIs) are commonly used in the clinical practice as an adjunctive tool in GERD diagnosis, the placebo effect is hard to be excluded. Thus objective detection of esophageal reflux is crucial in the confirmed diagnosis of GERD. Therefore, the ambulatory reflux monitoring is thought to be the "gold standard" for GERD diagnosis. When performing the reflux monitoring, the most reliable parameter is the acid exposure time (AET), which is defined as the time percentage of esophageal $\mathrm{pH}<4$ over the whole monitoring time (usually 24 hours). Patients who had AET $>4 \%$ is thought to be GERD. ${ }^{4}$ Although the ambulatory reflux test is thought to provide robust evidence for GERD, there are some conflicting scenarios in clinical practice which includes the poor response to acid suppression therapy in some patients with pathologic esophageal reflux using the cut-off of AET $>4 \%$. Thus the threshold for the pathologic esophageal reflux seems to be one of the determined factors that could influence the patients' response to acid suppressor. In order to better define GERD, experts in this field recently proposed in the Lyon consensus on GERD diagnostic test that AET $>6 \%$, instead of $4 \%$, should be conclusive evidence to GERD diagnosis based on the truth that more patients would show good response to PPIs with this new threshold. ${ }^{5}$

Although the endeavor to distinguish more "real" GERD patients was appreciated, there are some characteristic features in Chinese patients. It has been reported that the parietal cell in the stomachs of Asian population was less than that in the Western populations, so the acid secretion would differ between Asian and Western population. ${ }^{6}$ On the other hand, the proportion of patients with pathologic esophageal reflux was much lower in the Chinese population, ${ }^{7}$ only one-third of non-erosive reflux disease showed acid over-exposure. So we wondered whether the diagnostic threshold of the Lyon consensus should be adopted in the Chinese population. Therefore, we retrospectively reviewed the reflux monitoring parameters in our center in the attempt to investigate whether this diagnostic threshold is appropriate for GERD patients in China.

\section{Materials and Methods}

\section{Subjects}

Consecutive patients with typical heartburn and regurgitation who were referred to our hospital for esophageal function testing from December 1, 2010 to October 1, 2017 were screened. Patients with reflux symptoms for $>3$ months and with at least moderate symptom severity scores were included. All patients underwent upper endoscopy, esophageal high-resolution manometry (HRM) and 24-hour multichannel intraluminal impedance-pH (MII-pH) monitoring after at least 1 week of pharmacological washout. All patients signed an informed consent form prior to the examination. The Ethical Review Board of Sun Yat-sen University have approved the study (IRB No. [2019] 112). The study protocol was in line with the ethical guidelines of the 1975 Declaration of Helsinki.

Patients with AET greater than $4 \%$ were included, another group of patients from the same cohort with typical heartburn and reflux symptoms who had AET $<4 \%$ were also included as a control group. The following information, including demographic characteristics, upper gastrointestinal endoscopy, 24-hour MII-pH monitoring, HRM and their response to acid suppressed therapy were retrospectively analyzed. Patients who had history of esophagus or stomach surgery, upper gastrointestinal cancer, organic lesions observed in the upper gastrointestinal endoscopy including eosinophilic esophagitis, Barrett's esophagus, and peptic ulcers were excluded. Those who had major motility disorders on HRM were also excluded. Only patients who had continuous 8 weeks' therapy of PPIs were included.

All the included patients were divided into 3 groups according to their AET: AET $>6 \%$, AET between $4-6 \%$, and AET $<4 \%$. Furthermore, according to the patient's response to PPI therapy, they were divided into PPI responders and PPI non-responders. PPI responders refer to those whose PPI therapy were effective, otherwise they were PPI non-responders. When patients' symptoms improved for more than $50 \%$ compared with the baseline, PPI therapy was considered effective and these patients would be PPI responders. According to the Lyon consensus, those patients who had AET between 4-6\% were considered inconclusive GERD, so the adjunctive evidence including low mean nocturnal baseline impedance $(\mathrm{MNBI})(<2292 \Omega),{ }^{8}$ reflux episodes $>80$ and symptom association probability (SAP) positive were all added respectively in these patients to evaluate their therapeutic gain in PPI therapy. 


\section{Upper Gastrointestinal Endoscopy}

After overnight fasting, all included patients underwent upper gastrointestinal endoscopy in accordance with international guidelines. During the procedure, eosinophilic esophagitis was excluded by distal esophageal biopsy. The eosinophilia $>15$ per high-power field on biopsy is defined as eosinophilic esophagitis. Other lesions including upper gastrointestinal cancer, Barrett's esophagus and peptic ulcer patients were all excluded. The presence of hiatal hernia was recorded if recognized. According to the Los Angeles (LA) classification, we classified the included patients into (1) endoscopy negative or (2) reflux esophagitis.

\section{High-resolution Manometry}

Manometry parameters were recorded through HRM system (Medtronic Inc, Minneapolis, MN, USA). After calibration from $0 \mathrm{mmHg}$ to $300 \mathrm{mmHg}$, a solid-state manometric catheter with an outer diameter of $4 \mathrm{~mm}$, with 36 circumferential sensors spaced at $1 \mathrm{~cm}$ intervals, nasally fixed to the catheter and measured in the supine position of the patient. The baseline manometry and 10 single liquid swallows at 30 seconds were undertaken and recorded.

Two independent investigators manually analyzed all esophageal pressure topography using Manoview analysis software (Medtronic Inc), the following parameters were collected including esophagogastric junction (EGJ) inspiratory pressure, EGJ expiratory pressure, integrated relaxation pressure, distal contractile integral, distal latency, and esophageal motility classifications based on the Chicago criteria. ${ }^{9}$ EGJ morphology was described for all the included patients according to the Chicago classification: with type I EGJ morphology, there is complete overlap of crural diaphragm and lower esophageal sphincter (LES) components with single peak on the spatial pressure variation plot; with type II EGJ morphology, there is double-peaked pressure zone with the interpeak nadir pressure greater than gastric pressure and a separation of 1-2 cm between peaks; and with type III EGJ morphology, the inspiratory spatial pressure variation plot exhibits $>2 \mathrm{~cm}$ separation between the LES and crural diaphragm peaks with the nadir pressure between them equal to or less than gastric pressure.'

\section{Twenty-four Ambulatory Multichannel Intraluminal Impedance-pH Monitoring}

We performed 24-hour MII-pH monitoring using an ambulatory MII-pH monitoring system (Sandhill Scientific Inc, Highland Ranch, CO, USA). After calibrating in buffer solutions at $\mathrm{pH}$ 4.0 and 7.0, the catheter (ZAI-BS-01; Sandhill Scientific Inc) was placed through the nasal cavity with the $\mathrm{pH}$ electrode located $5 \mathrm{~cm}$ above the LES, and 6 impedance channels were located in 3, 5, 7, 9, 15 , and $17 \mathrm{~cm}$ proximal to the LES. During monitoring, postures, meals and symptoms were recorded. Furthermore, they were encouraged to keep their daily life as usual during the monitoring.

Two researchers manually analyzed the data using Bioview Analysis Software (Bioview Analysis; Sandhill Scientific Inc). Meal periods were not included in the analysis. The following parameters were collected including: (1) AET, (2) reflux episode, (3) proximal reflux episode, (4) gas associated reflux episode, (5) SAP (positive if $\geq 95 \%$ ), and (6) MNBI (it was calculated by extracting baseline impedance values at impedance channel across stable nocturnal 10-minute periods [at or around $1 \mathrm{AM}, 2 \mathrm{AM}$, and $3 \mathrm{AM}$ ] avoiding reflux events or swallows. The values from the 3 -time periods were averaged to yield the MNBI).

\section{Statistical Methods}

Data with normal distribution were presented as mean \pm standard deviation, while others were presented as the median (25th, 75th). ANOVA or Kruskal-Wallis test was used to for multiple group comparison, and Mann-Whitney $U$ was used for 2 groups' comparison. We compared categorical data using the chi-squared test. Statistical analysis was done using the SPSS version 20.0 (IBM

Table 1. Demographic Characteristics Compared Among Groups Based on Acid Exposure Time

\begin{tabular}{|c|c|c|c|c|}
\hline Demographic characteristics & $\mathrm{AET}>6 \%(\mathrm{n}=113)$ & AET4-6\% $(\mathrm{n}=38)$ & $\mathrm{AET}<4 \%(\mathrm{n}=144)$ & $P$-value \\
\hline Male gender & $75(66.4)$ & $27(71.1)$ & $73(50.7)$ & $0.011^{\mathrm{a}}$ \\
\hline Age (yr) & $49.55 \pm 14.07$ & $46.00 \pm 14.05$ & $43.26 \pm 13.34$ & $0.002^{\mathrm{a}}$ \\
\hline $\operatorname{BMI}\left(\mathrm{kg} / \mathrm{m}^{2}\right)$ & $23.10 \pm 3.26$ & $23.32 \pm 3.11$ & $21.53 \pm 3.05$ & $<0.001^{\mathrm{a}}$ \\
\hline \multicolumn{5}{|l|}{$\mathrm{RE}$} \\
\hline LA grade A & $12(10.6)$ & $3(7.9)$ & $9(6.2)$ & $<0.001^{\mathrm{a}}$ \\
\hline LA grade B & $30(26.5)$ & $10(26.3)$ & $9(6.2)$ & $<0.001^{\mathrm{a}}$ \\
\hline
\end{tabular}

AET, acid exposure time; BMI, body mass index; RE, reflux esophagitis; LA, Los Angeles classification.

Data presented as mean $\pm \mathrm{SD}$ or $\mathrm{n}(\%)$.

${ }^{a} P<0.05$ when patients with AET $>6 \%$ compared with AET $<4 \%$. 
Corp, Armonk, NY, USA). A $P$-value $(<0.05)$ with 2 tails was considered statistically significant.

\section{Results}

In total, 151 patients (102 males, age $48.66 \pm 14.11$ years) were finally included, among them, 113 patients were with AET $>6 \%$ (74.9\%), and the rest of the 38 patients with AET between 4-6\% (25.2\%). Another group of patients $(\mathrm{n}=144,73$ males, age $43.26 \pm 13.34$ years) with AET $<4 \%$ were also included as controls. The demographic characteristics are listed in Table 1, patients with AET $>6 \%$ or AET 4-6\% were similar in terms of age, gender ratio, body mass index and the proportion of reflux esophagitis. While in patients with AET $<4 \%$, there were more females, younger patients with lower body mass index, and less patients with esophagitis.

\section{Comparison of Manometry Parameters}

The comparison of the HRM parameters between patients with AET $>6 \%$ and AET $4-6 \%$ are listed in Table 2. The AET $>6 \%$ and AET 4-6\% patients showed higher EGJ inspiratory pressure and lower integrated relaxation pressure than the AET $<4 \%$ patients. No statistical differences were found in the distal latency, EGJ expiratory pressure, and distal contractile

Table 2. High-resolution Manometry Parameters Compared Among Groups Based on Acid Exposure Time

\begin{tabular}{|c|c|c|c|c|}
\hline Parameters & $\operatorname{AET}>6 \%(\mathrm{n}=113)$ & AET $4-6 \%(n=38)$ & $\mathrm{AET}<4 \%(\mathrm{n}=144)$ & $P$-value \\
\hline \multicolumn{5}{|l|}{ EGJ parameters } \\
\hline EGJ inspiratory pressure $(\mathrm{mmHg})$ & $17.4(12.3-24.0)$ & $15.6(12.4-18.6)$ & $18.0(14.1-24.8)$ & $0.029^{\mathrm{a}}$ \\
\hline EGJ expiratory pressure (mmHg) & $11.0(6.5-17.0)$ & $9.2(4.1-13.0)$ & $11.0(8.0-16.0)$ & 0.059 \\
\hline EGJ-CI (mmHg·sec $\cdot \mathrm{cm})$ & $35.6(13.0-91.0)$ & $20.3(7.4-97.1)$ & $54.0(16.3-117.4)$ & 0.239 \\
\hline IRP-4s (mmHg) & $7.1(4.7-10.0)$ & $5.9(4.6-7.0)$ & $8.1(5.7-10.0)$ & $0.001^{\mathrm{a}}$ \\
\hline \multicolumn{5}{|l|}{ EGJ morphology } \\
\hline Type I & $98.6 \%$ & $100.0 \%$ & $98.2 \%$ & 0.716 \\
\hline Type II & $1.4 \%$ & $0.0 \%$ & $1.8 \%$ & 0.716 \\
\hline \multicolumn{5}{|l|}{ Peristalsis parameters } \\
\hline $\mathrm{DL}(\mathrm{sec})$ & $6.4(5.7-7.4)$ & $6.6(5.7-7.2)$ & $6.3(5.6-7.0)$ & 0.602 \\
\hline $\mathrm{DCI}(\mathrm{mmHg} \cdot \mathrm{sec} \cdot \mathrm{cm})$ & $279.0(154.0-665.0)$ & $356.5(205.0-622.0)$ & $281.5(161.0-589.0)$ & 0.471 \\
\hline \multicolumn{5}{|l|}{ Chicago classification } \\
\hline IEM & $31.6 \%$ & $40.0 \%$ & $27.7 \%$ & 0.403 \\
\hline Normal & $68.4 \%$ & $60.0 \%$ & $72.3 \%$ & 0.403 \\
\hline
\end{tabular}

AET, acid exposure time; EGJ, esophagogastric junction; EGJ-CI, esophagogastric junction contractile integral; IRP-4s, 4-second integrated relaxation pressure; DL, distal latency; DCI, distal contractile integral; IEM, ineffective esophageal motility.

Data presented as median (25th, 75th).

${ }^{a} \mathrm{P}<0.05$ when patients with AET $>4 \%$ compared with AET $<4 \%$.

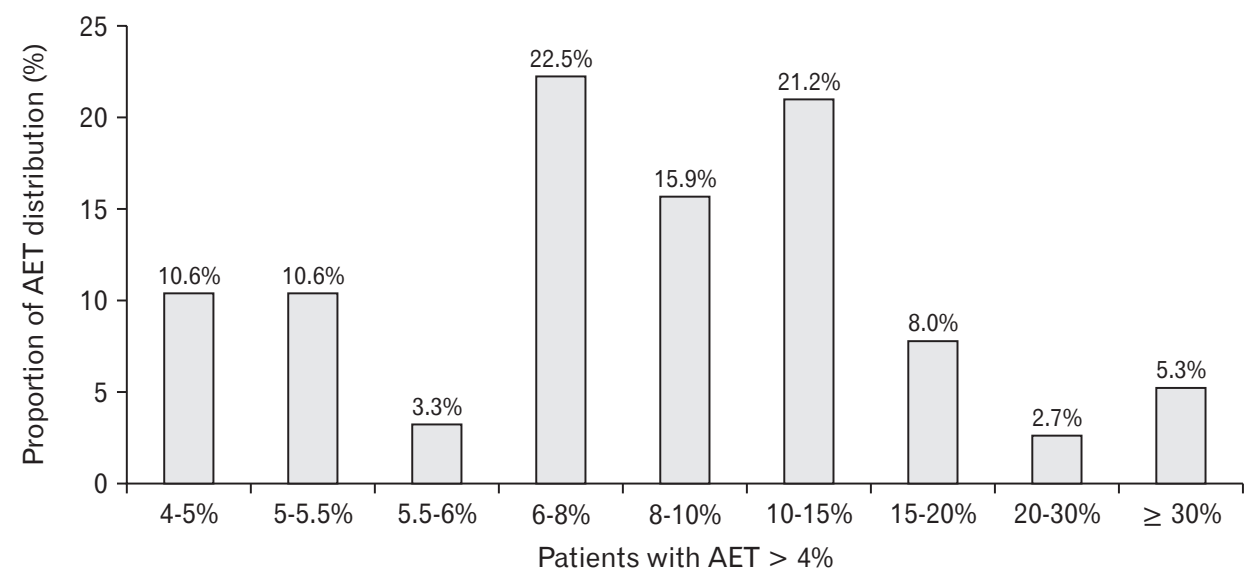

Figure 1. The acid exposure time (AET) distribution of patients with AET $>4 \%$. $24.5 \%$ of these patients were within the domain of AET $<6 \%$, the rest of them had $\mathrm{AET}>6 \%$. 
integral. EGJ-contractile integral values for patients with AET $>6 \%$ and AET $4-6 \%$ appear to be lower than those with AET $<4 \%$, but the difference did not reach statistical difference (Table 2).

\section{Twenty-four-hour Multichannel Intraluminal Impedance-pH Monitoring}

The AET distribution within patients with AET $>4 \%$ are shown in Figure 1, and $24.5 \%$ of these patients were within the domain of AET $<6 \%$, the rest of them had AET $>6 \%$.

The reflux parameters are listed in Table 3 , there were no differences between these 2 groups. The PPI response rates of patients were $64.6 \%$ and $63.2 \%$ in patients with AET $>6 \%$ and AET 4-6\% respectively (Fig. 2). No statistical difference was found in PPI therapy between these 2 groups. In addition, we also compared the $\mathrm{pH}$ monitoring parameters according to PPI therapy, and there was still no difference between them.

According to the Lyon consensus, AET between 4-6\% was categorized as inconclusive GERD, thus adjunctive evidence would be necessary. So we added the adjunctive evidence including low MNBI $(<2292 \Omega)$, reflux episodes $>80$ and SAP $>95 \%$ in patients with 4-6\% and compared them with patients with AET $>6 \%$ (Table 4). When the patients with AET $>6 \%$ were compared to the patients with AET 4-6\% plus reflux episodes $>80$, there were more reflux episodes in the latter group, otherwise there was no difference among the other esophageal $\mathrm{pH}$ metrics. Similarly, there was no difference between the patients with AET $>6 \%$ and AET $4-6 \%$ plus either low MNBI or SAP > 95\%. Most importantly, when the efficacy of PPI therapy was compared, no therapeutic gain was obtained even if the adjunctive evidence was added in patients with AET 4-6\%. No statistical difference was found in PPI therapy between these groups (Fig. 3).

\section{Symptom Association Probability}

Among patients with AET > 6\%, 54 (47.8\%) were SAP positive, while 16 (42.1\%) were SAP positive among patients with AET 4-6\%. Among patients with AET < 4\% ( $\mathrm{n}=144), 53$ (36.8\%) were SAP positive. The motility and reflux profile of these patients with SAP positive in each group was compared. No statistical difference was found among them.

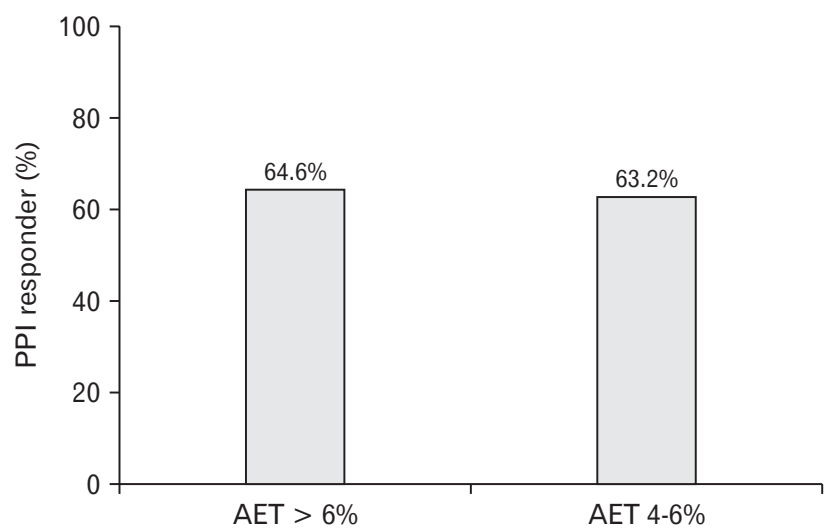

Figure 2. The efficacy of proton pump inhibitor (PPI) therapy in patients with acid exposure time $(\mathrm{AET})>4 \%$. The PPI response rates of patients were $64.6 \%$ and $63.2 \%$ in patients with AET $>6 \%$ and AET 4-6\%, respectively $(P=0.872)$.

Table 3. Twenty-four-hour Multichannel Intraluminal Impedance-pH Monitoring Parameters Compared Among Groups Based on Acid Exposure Time

\begin{tabular}{|c|c|c|c|c|c|}
\hline Parameters & $\mathrm{AET}>6 \%(\mathrm{n}=113)$ & AET $4-6 \%(n=38)$ & $P$-value & $\mathrm{AET}<4 \%(\mathrm{n}=144)$ & $P$-value \\
\hline \multicolumn{6}{|l|}{ Reflux episode } \\
\hline Acid & $41(31-56)$ & $40(28-49)$ & 0.440 & $11(3-24)$ & $0.000^{\mathrm{b}}$ \\
\hline Weakly acidic & $16(8-26)$ & $19(12-30)$ & $0.038^{\mathrm{a}}$ & $18(11-27)$ & 0.073 \\
\hline Weakly alkaline & $0(0-1)$ & $0(0-1)$ & 0.299 & $0(0-2)$ & 0.299 \\
\hline All reflux & $57(45-80)$ & $63(49-72)$ & 0.777 & $37(23-50)$ & $0.000^{\mathrm{b}}$ \\
\hline \multicolumn{6}{|c|}{ Proximal reflux episode } \\
\hline Acid & $28(17-35)$ & $23(13-31)$ & 0.426 & $5(1-13)$ & $0.000^{\mathrm{b}}$ \\
\hline Weakly acidic & $7(3-11)$ & $7(6-15)$ & 0.116 & $7(4-11)$ & 0.274 \\
\hline Weakly alkaline & $0(0-0)$ & $0(0-0)$ & 0.605 & $0(0-1)$ & 0.605 \\
\hline All reflux & $36(22-43)$ & $33(19-45)$ & 0.827 & $14(8-23)$ & $<0.001^{\mathrm{b}}$ \\
\hline $\operatorname{MNBI}(\Omega)$ & $940(648-1873)$ & $1344(880-2204)$ & 0.338 & $2815(1965-3438)$ & $<0.001^{\mathrm{b}}$ \\
\hline
\end{tabular}

MNBI, mean nocturnal baseline impedance; AET, acid exposure time.

Data presented as median (25th, 75 th).

${ }^{a} P<0.05$ when patients with AET $>6 \%$ compared with AET 4-6\%.

${ }^{\mathrm{b}} \mathrm{P}<0.05$ when patients with AET $>4 \%$ compared with AET $<4 \%$. 
Table 4. Twenty-four-hour Multichannel Intraluminal Impedance-pH Monitoring Parameters Compared Among Groups Based on Acid Exposure Time With Additional Adjunctive Evidence Proposed in Lyon Consensus

\begin{tabular}{|c|c|c|c|c|c|c|c|}
\hline Parameters & $\begin{array}{c}\text { AET > 6\% } \\
(\mathrm{n}=113)\end{array}$ & $\begin{array}{c}\text { AET } 4-6 \% \\
\text { and low MNBI } \\
(\mathrm{n}=25)\end{array}$ & $P$-value & $\begin{array}{c}\text { AET } 4-6 \% \\
\text { and reflux episodes } \\
>80(\mathrm{n}=9)\end{array}$ & $P$-value & $\begin{array}{l}\text { AET } 4-6 \% \\
\text { and SAP positive } \\
(\mathrm{n}=16)\end{array}$ & $P$-value \\
\hline \multicolumn{8}{|l|}{ Reflux episode } \\
\hline Acid & $41(31-56)$ & $39(26-49)$ & $>0.999$ & $63(57-79)$ & $0.012^{\mathrm{a}}$ & $30(42-56)$ & $>0.999$ \\
\hline Weakly acidic & $16(8-26)$ & $17(10-28)$ & 0.886 & $33(17-36)$ & $0.039^{\mathrm{a}}$ & $21(12-29)$ & 0.466 \\
\hline Weakly alkaline & $0(0-1)$ & $0(0-1)$ & 0.473 & $0(0-2)$ & 0.856 & $0(0-1)$ & 0.591 \\
\hline All reflux & $57(45-80)$ & $60(49-72)$ & $>0.999$ & $93(87-112)$ & $0.001^{\mathrm{a}}$ & $70(51-72)$ & $>0.999$ \\
\hline \multicolumn{8}{|c|}{ Proximal reflux episode } \\
\hline Acid & $28(17-35)$ & $23(15-33)$ & 0.537 & $30(23-50)$ & 0.082 & $28(18-36)$ & 0.604 \\
\hline Weakly acidic & $7(3-11)$ & $8(5-16)$ & 0.613 & $8(7-17)$ & 0.072 & $8(6-18)$ & 0.129 \\
\hline Weakly alkaline & $0(0-0)$ & $0(0-0)$ & 0.132 & $0(0-1)$ & 0.341 & $0(0-0)$ & 0.801 \\
\hline All reflux & $36(22-43)$ & $37(23-48)$ & 0.078 & $51(33-63)$ & 0.073 & $41(26-51)$ & 0.218 \\
\hline MNBI & $940(648-1873)$ & $1284(811-1409)$ & 0.778 & $1284(545-1431)$ & 0.824 & $1312(812-1431)$ & 0.862 \\
\hline PPI responder $(\%)$ & $73(64.6)$ & $17(68)$ & 0.747 & $2(22.2)$ & $0.012^{\mathrm{a}}$ & $11(68.8)$ & 0.745 \\
\hline
\end{tabular}

AET, acid exposure time; MNBI, mean nocturnal baseline impedance; SAP, symptom association probability, PPI, proton pump inhibitor. Data presented as median (25th, 75th) or $\mathrm{n}(\%)$.

${ }^{a} \mathrm{P}<0.05$ when patients with AET $>6 \%$ compared with AET 4-6\% and reflux episodes $>80$.

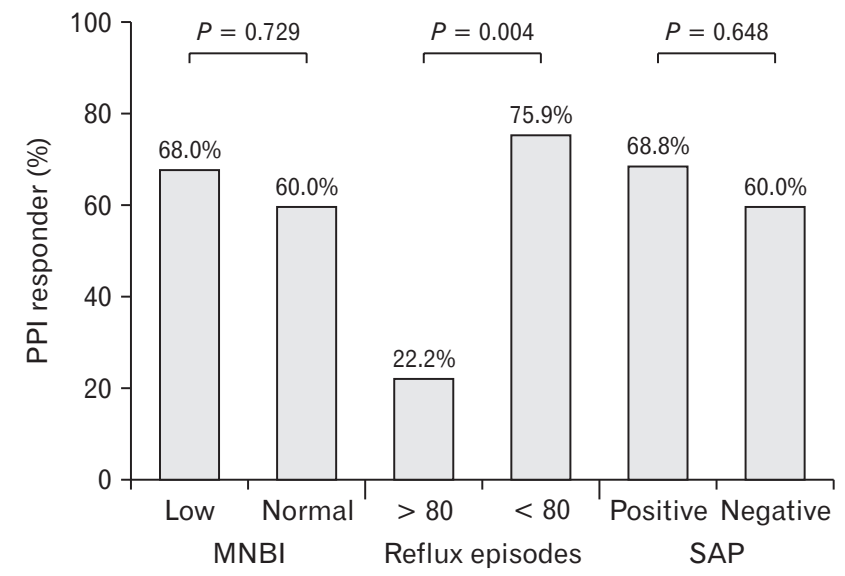

Figure 3. Efficacy of proton pump inhibitor (PPI) therapy in patients with acid exposure time (AET) 4-6\% with different adjunctive evidence added. The PPI response rates of patients were $68.0 \%$ and $60.0 \%$ in patients with low mean nocturnal baseline impedance (MNBI) and normal MNBI respectively $(P=0.729)$. The PPI response rates of patients were $22.2 \%$ and $75.9 \%$ in patients with reflux episodes $>80$ and reflux episodes $<80$, respectively $(P=0.004)$. The PPI response rates of patients were $68.8 \%$ and $60.0 \%$ in patients with symptom association probability (SAP) positive and SAP negative, respectively $(P=0.648)$.

\section{Discussion}

The Lyon consensus proposed that advanced grades of erosive esophagitis (LA grades C and D), long-segment Barrett's mucosa or peptic strictures on endoscopy or distal esophageal AET $>6 \%$ on ambulatory $\mathrm{pH}$ or $\mathrm{pH}$-impedance monitoring is the conclusive evidence for reflux. ${ }^{1}$ One of the purposes of the updated criteria was to improve the diagnosis of GERD, thus inappropriate use of acid-suppressors would be avoided. However, less acid burden in Chinese GERD patients has been reported in a previous study. ${ }^{7}$ So, using the stricter criteria to diagnose GERD patients in the Chinese population might result in far less GERD patients being diagnosed. In order to see whether this updated criterion was appropriate in the Chinese population with less acid burden, we respectively reviewed those GERD patients defined by the previous criteria of AET > $4 \%$, and compared the reflux and manometric profile between patients with AET 4-6\% and $>6 \%$. It turned out that using the cutoff value $6 \%$ for AET did decrease the diagnostic rate of GERD patients, while response rate to PPI therapy did not increase with the threshold for GERD raised.

The need was still unmet in terms of diagnosis of GERD since the current diagnostic methods, including symptom questionnaire, upper endoscopy, reflux monitoring, and pepsin test, had some defects. For example, questionnaires could not exclude patients with functional esophageal symptoms and upper endoscopy could only recognize patients with mucosal injury. That is why the Lyon consensus tried to strengthen the diagnosis criteria for GERD. The purpose of updated criteria was to better define GERD and improve the clinical outcome. Increased threshold should be able to recognize the "real" GERD patients, thus increase the efficacy of PPI therapy among these patients. 
AET, defined by the time percentage of esophageal $\mathrm{pH}$ less than 4 during 24-hour $\mathrm{pH}$ monitoring, is recognized as the primary parameter in ambulatory $\mathrm{pH}$ monitoring. The original threshold for AET in catheter-based $\mathrm{pH}$ monitoring of PPI was set to $4 \%$ because the AET among asymptomatic controls and patients with occasional ( $<2$ times/month) heartburn symptoms ranged from $3.9 \%$ to $7.2 \% .^{10}$ On the other hand, the average total AET of patients with erosive esophagitis was 9.6-27.6\%, ${ }^{11}$ thus the Lyon consensus group considered AET $>6 \%$ to be abnormal. Previously, studies have suggested that elevated AET could predict a positive response to PPI trials. ${ }^{12,13}$ Thus, increasing the AET threshold to define GERD should inevitably improve the efficacy of PPI therapy. However, in the current study, the response rate of PPI therapy among the patients with AET $>6 \%$ was $64.6 \%$, while the corresponding rate among patients with AET between 4-6\% was $63.2 \%$. No significant difference was detected between these 2 groups. Thus increasing the cut-off value of AET to 6\% seemed unable to improve the clinical outcome. So applying the new threshold of AET should be cautious if the endpoint was to improve the therapeutic outcome.

According to the Lyon consensus, those patients who had AET between 4-6\% were defined as inconclusive GERD, who need adjunctive evidence to make a confirmed diagnosis. We also analyzed the MNBI, total reflux episode, and SAP which provided the adjunctive evidence for GERD. When these additional evidences were included, the proportion of confirmed GERD varied with the evidence provided. For example, 25 out of 38 inconclusive GERD would be confirmed if MNBI $<2292 \Omega$ was applied. However, only 9 out of 38 would be confirmed if the number of total reflux episodes was applied. The reflux parameters and PPI response remained similar even when the additional evidence was added, so the adjunctive evidence of these included inconclusive GERD patients did not help to improve the therapeutic outcome. Although GERD is a common disease in a gastrointestinal clinic, confident diagnosis of GERD is difficult to reach and multiple methods are needed to confirm the diagnosis.

The current study also focused on the motility characteristic of different groups of patients. Apparently, the patients with AET > $4 \%$ showed much weaker anti-reflux barrier compared with those of AET $<4 \%$ group in terms EGJ pressure and EGJ morphology. Previous studies have confirmed that GERD patients with acid over-exposure were with injury anti-reflux barrier. ${ }^{14}$ However, the motility profile were similar between patients with AET $>6 \%$ and 4-6\%. It has been reported that GERD patients with much higher levels of acid exposure did not have worse esophageal peristalsis, ${ }^{14}$ so using only AET as a grouping factor was not appropriate.

There are some limitations in the current study. The proportion of patients with RE were all with LA-grade $\mathrm{A}$ and $\mathrm{B}$, which were recognized as inconclusive evidence of GERD according to the Lyon consensus. However, according to our previous study, ${ }^{15} \mathrm{Chi}-$ nese GERD patients were with less acid burden and about $95 \%$ of erosive esophagitis patients were with LA-grade A and B. Thus, the proportion of esophagitis patients actually reflected the real distribution of esophagitis in Chinese GERD patients. On the other hand, the ambulatory reflux monitoring has day-to-day variability. Some patients might have more esophageal secondary peristalsis due to the throat discomfort caused by the intubation, which made less acid exposure being detected. Thirdly, this was a retrospective study, and the PPI response of all the included patients were recorded according to their medical charts, which would be one source of bias. What is more, only the distal biopsy was performed instead of both proximal and distal biopsies during the examination, the number and position of biopsy samples could impact diagnostic ability. Finally, the sample size of the current study was relatively small, which would undermine the power of the study. A multicenter study with larger sample size is necessary to confirm the hypothesis.

In conclusion, increasing the AET threshold in the Lyon consensus was not able to improve the clinical outcome considering that efficacy of PPI therapy was similar in patients with AET > $6 \%$ and $4-6 \%$. So the diagnosis of GERD should rely on not only AET but multiple combined methods.

Financial support: The study was supported by grants from the National Natural Science Foundation of China (81770544 and 81970479).

\section{Conflicts of interest: None}

Author contributions: Yuqing Lin: study concept and design, acquisition of data, analysis and interpretation of data, and drafting and finalizing the manuscript; Yuwen Li: analysis and interpretation of data, and reviewing and editing the manuscript; Mengya Liang, Niandi Tan, Mengyu Zhang, and Songfeng Chen: acquisition of data; and Yinglian Xiao: study concept and design, finalizing the manuscript, and guarantee of the study.

\section{References}

1. Gyawali CP, Kahrilas PJ, Savarino E, et al. Modern diagnosis of GERD: the Lyon Consensus. Gut 2018;67:1351-1362.

2. Roman S, Keefer L, Imam H, et al. Majority of symptoms in esophageal 
reflux PPI non-responders are not related to reflux. Neurogastroenterol Motil 2015;27:1667-1674.

3. Weijenborg PW, Smout AJ, Verseijden C, et al. Hypersensitivity to acid is associated with impaired esophageal mucosal integrity in patients with gastroesophageal reflux disease with and without esophagitis. Am J Physiol Gastrointest Liver Physiol 2014;307:G323-G329.

4. Carlson DA, Kathpalia P, Craft J, et al. The relationship between esophageal acid exposure and the esophageal response to volumetric distention. Neurogastroenterol Motil 2018;30:e13240.

5. Lundell LR, Dent J, Bennett JR, et al. Endoscopic assessment of oesophagitis: clinical and functional correlates and further validation of the Los Angeles classification. Gut 1999;45:172-180.

6. Lam SK. Differences in peptic ulcer between East and West. Baillieres Best Pract Res Clin Gastroenterol 2000;14:41-52.

7. Nikaki K, Woodland P, Sifrim D. Adult and paediatric GERD: diagnosis, phenotypes and avoidance of excess treatments. Nat Rev Gastroenterol Hepatol 2016;13:529-542.

8. Patel A, Wang D, Sainani N, Sayuk GS, Gyawali CP. Distal mean nocturnal baseline impedance on $\mathrm{pH}$-impedance monitoring predicts reflux burden and symptomatic outcome in gastro-oesophageal reflux disease. Aliment Pharmacol Ther 2016;44:890-898.

9. Kahrilas PJ, Bredenoord AJ, Fox M, et al. The Chicago Classifica- tion of esophageal motility disorders, v3.0. Neurogastroenterol Motil 2015;27:160-174.

10. Masclee AA, de Best AC, de Graaf R, Cluysenaer OJ, Jansen JB. Ambulatory 24-hour $\mathrm{pH}$-metry in the diagnosis of gastroesophageal reflux disease. Determination of criteria and relation to endoscopy. Scand J Gastroenterol 1990;25:225-230.

11. Mattioli S, Pilotti V, Spangaro M, et al. Reliability of 24-hour home esophageal $\mathrm{pH}$ monitoring in diagnosis of gastroesophageal reflux. Dig Dis Sci 1989;34:71-78.

12. Ribolsi M, Savarino E, De Bortoli N, et al. Reflux pattern and role of impedance-pH variables in predicting PPI response in patients with suspected GERD-related chronic cough. Aliment Pharmacol Ther 2014;40:966-973.

13. Wang AJ, Wang H, Xu L, et al. Predictors of clinical response of acid suppression in Chinese patients with gastroesophageal reflux disease. Dig Liver Dis 2013;45:296-300.

14. Xie C, Wang J, Li Y, et al. Esophagogastric junction contractility integral reflect the anti-reflux barrier dysfunction in patients with gastroesophageal reflux disease. J Neurogastroenterol Motil 2017;23:27-33.

15. Zhang M, Tan N, Li Y, Chen M, Xiao Y. Esophageal physiologic profiles within erosive esophagitis in China: predominantly low-grade esophagitis with low reflux burden. Neurogastroenterol Motil 2019;31:e13702. 